\title{
Minimizing rework costs in multistage production processes by modifying quality specification limits
}

\author{
David de-Felipe \\ Bayerische Motoren Werke AG, \\ BMW AG \\ Munich, Germany \\ David.de-Felipe@bmw.de
}

\author{
Ernest Benedito \\ Department of Management and Institute of Industrial and \\ Control Engineering \\ Universitat Politècnica de Catalunya, UPC \\ Barcelona, Spain \\ ernest.benedito@upc.edu
}

\begin{abstract}
Multistage production processes are becoming more important in the industry to ensure levels of flexibility, efficiency and modularity. Thus, the way in which companies define optimal production parameters related to production costs and quality must be adapted to this reality. In this paper we introduce a multi-response optimization (MRO) model for a two stage production process. The model gives first stage quality specification limits which minimize the rework costs caused by the nonconforming parts of the whole process. The proposed model is applied to an example based on a production process of the automotive industry. The benefits of the model are evaluated by comparing the capability and the rework costs of the multistage production process before and after the optimization.
\end{abstract}

Keywords-Cyber-Physical Systems, multistage production processes, online operation conditions, rework costs, production quality.

\section{INTRODUCTION}

Cyber-Physical Systems (CPSs) are the new generation of engineered systems combining computational and physical elements [1] and are the base of the Industry 4.0 concept [2]. In this context, flexibility, adaptability, modularity, autonomy, reliability, safety and efficiency are rated as the key features of CPSs [3]. Hereafter, we focus on efficiency and adaptability of CPSs.

With regards to efficiency of CPSs, achieving optimal design production parameters is possible by following Taguchi's principles and theories (Taguchi et al. [4]), who's main objective is to obtain the optimal levels of many design factors of a production process which allow achieving the minimal production costs while assuring a certain level of quality.

Taking adaptability into account, trying to stabilize production costs and production quality while CPSs are running under different conditions is also a great challenge. While CPSs are running online, multiple parameters vary under different operation conditions resulting in a deterioration of the performance of the process [5]. For example, tool wear or tool breakage can appear, which have the effect of moving the product characteristics away from the target values. This fact results in higher nonconforming rates and, consequently, in higher rework costs -i.e. the costs that a company assume when describing outputs as nonconforming.

Unfortunately, it is not easy to modify production parameters online due to different reasons such as lack of access to parameter design factors once processes are already running, or the high time that is required to reach agreements related to parameters modifications. Furthermore, the recalibration of machines to the initial design configuration is expensive and time consuming. However, in CPSs, such as multistage production processes, other production parameters can be easily modified. This can help to minimize production costs while machines cannot be recalibrated.

In multistage production processes, the quality of the end products depends on the specification limits used in the previous stages, among other production parameters. Thus, these parameters have an influence on the proportion of conforming and nonconforming parts in the next sub-processes. Taking this into account, it is possible to influence the quality of the products at the end of the process by modifying the specification limits in the previous stages.

The purpose of this paper is to present a multi-response optimization (MRO) model valid in multivariate and multistage production processes that minimizes rework costs. With this model, we suggest obtaining the optimal configuration of the specification limits in the first sub-process of a two stage production process. This MRO model assumes that the measures of the outputs follow a (multi-) normal distribution. The proposed model is applied to an example based on a production process within the automotive industry. The value of the model is evaluated by comparing the capability and the rework costs of the multistage production process before and after the optimization.

The rest of the paper has the following structure: In section II, MRO models and univariate and multivariate process capability indices (PCIs) existing in the literature are presented. In section III, the new MRO model is presented. In section IV the model is applied and discussed in an example based on the automotive industry. The paper concludes in section $\mathrm{V}$. 


\section{MRO MODELS AND PCIS}

In this section we introduce some MRO models to solve MRO problems. Furthermore, we also introduce two univariate PCIs (the $\mathrm{C}_{\mathrm{p}}$ and the $\mathrm{C}_{\mathrm{pk}}$ ) and a multivariate PCI (the $\mathrm{MC}_{\mathrm{pk}}$ ).

In MRO problems, it is assumed that $n$ product characteristics, namely performance measures, $\left(\mathrm{Y}_{1}, \mathrm{Y}_{2}, \ldots, \mathrm{Y}_{\mathrm{n}}\right)$ depend on $m$ factors $\left(\mathrm{X}_{1}, \mathrm{X}_{2}, \ldots, \mathrm{X}_{\mathrm{m}}\right)$ through a known function $f \quad$ (i.e. $\quad \mathrm{Y}_{1}=f\left(X_{1}, X_{2}, \ldots, X_{m}\right)$, $\left.\mathrm{Y}_{2}=f\left(X_{1}, X_{2}, \ldots, X_{m}\right), \ldots, \quad \mathrm{Y}_{\mathrm{n}}=f\left(X_{1}, X_{2}, \ldots, X_{m}\right)\right)$. Unfortunately, in MRO problems, maximizing or minimizing each relevant product characteristic individually does not always deal with obtaining the global optimum. Taking this into account, many MRO models have been presented in the literature. Hereafter, some MRO models to solve MRO problems are discussed.

MRO models can be classified into two different groups: Methods based on single responses approaches and methods based on constrained optimization approaches. Since the MRO presented in this article is a single response approach, we will focus our attention on this technique.

The methods based on single responses include all approaches that group all product characteristics into a single response. In this group we can find methods including the desirability function (Harrington [6], Derringer and Suich [7], del Castillo et al. [8], Kim and Lin [9] and $\mathrm{Wu}$ and Hamada [10]), the quadratic loss function (Pignatiello [11], Vining [12], Tsui [13], Ribeiro et al. [14] and Ko et al. [15]), the signal-tonoise rate (Tong et al. [16], Liao [17], Tong et al. [18] and Gauri and Chakraboroty [19]) a single response based on the proportion of conforming parts (Chiao and Hamada [20]), a Bayesian approach (Peterson [21]), a generalized distance function (Khuri and Conlon [22]) and single response methods based on PCIs (Lee and Yum [23], Awad and Kovach [24], Amiri et al.[25] and Bera and Mukherjee [26]) among others.

PCIs are statistic coefficients used in the industry to quantify how well a process can meet process requirements by relating the variability of the measures of a product characteristic with the admissible one. The $C_{p}(1)$ and the $C_{p k}$ (2) indices in Kane [27] are univariate PCIs extendedly accepted and used in the industry which can be calculated with the following expressions:

$$
\begin{gathered}
\mathrm{C}_{\mathrm{p}}=(\mathrm{USL}-\mathrm{LSL}) / 6 \mathrm{~s} \\
\mathrm{C}_{\mathrm{pk}}=\operatorname{Min}\{(\mathrm{USL}-\overline{\mathrm{x}}) / 3 \mathrm{~s} ;(\overline{\mathrm{x}}-\mathrm{LSL}) / 3 \mathrm{~s}\}
\end{gathered}
$$

In (1) and (2) $s$ is the standard deviation of the measures and $\bar{x}$ represents the mean value of the measures. LSL and USL are the lower and upper specification limits.

Multivariate PCIs have been recently introduced to describe the entire production variability derived from the multivariate case. Thanks to multivariate PCIs, the capability of a process with $v$ product characteristics can be summarized with one single index. Among all the multivariate PCIs existing in the literature, we suggest using the $\mathrm{MC}_{\mathrm{pk}}$ (3) in de-Felipe et al. [28].

$$
\mathrm{MC}_{\mathrm{pk}}=-1 / 3 \Phi^{-1}\left(\mathrm{NCP}_{\text {crit }}\right)
$$

This multivariate PCI is a function of the expected total proportion of nonconforming parts in the most critical direction and is obtained through the cumulative distribution function $(\Phi)$ of the standard normal distribution $N(0,1)$.

\section{Multi-Response Optimization ModeL}

In this section we introduce an optimization model that minimize rework costs by achieving the optimal position of the specification limits in the first sub-process of multistage production processes. In this model it is assumed that the specification limits in the last sub-process of the multistage production process are fixed.

The MRO model introduced in this article is based on the model presented in Chiao and Hamada [20] but differs from it in three aspects: First, the model uses the modularity derived from multistage production processes by considering each stage individually and afterwards the whole multistage process as a global system. Second, in the presented model, rework costs are taken into account. Third, in the presented model, the optimization variables are the specification limits.

Before introducing the model, we want to make clear that we assume that the measures follow a $v$-variate normal distribution, with mean vector $\boldsymbol{\mu}$, and variance-covariance matrix $\sum$. Then, the probability density function is described with (4); and then, the probability that a measure is within a region $R$ can be calculated as the integral of (4) within $R$.

$$
f\left(x, \boldsymbol{\mu}, \sum, v\right)=\frac{1}{\sqrt{\left|\sum\right|(2 \pi)^{v}}} e^{-\frac{1}{2}(\boldsymbol{x}-\boldsymbol{\mu})^{\prime} \sum^{-1}(\boldsymbol{x}-\boldsymbol{\mu})}
$$

Imagine a multistage production process -like the one represented in Fig. 1- composed of two sub-processes $\left(\mathrm{SP}_{1}\right.$ and $\mathrm{SP}_{2}$ ) and their respective measuring stations and quality tests.

After $\mathrm{SP}_{1}$ the quality of the outputs is evaluated by analyzing $m$ product characteristics: $\mathrm{PC}_{\mathrm{SP} 1,1}, \ldots, \mathrm{PC}_{\mathrm{SP} 1, \mathrm{~m}}$. For each product characteristic $i$ of $\mathrm{SP}_{1}\left(\mathrm{PC}_{\mathrm{SP} 1, \mathrm{i}}, i \in[1, m] ; \mathrm{i} \in \mathbb{N}\right)$, the measured values of the $m$ product characteristics must be within $\mathrm{LSL}_{\mathrm{SP}_{1, \mathrm{i}}}$ and $\mathrm{USL}_{\mathrm{SP}_{1, \mathrm{i}}}$. With the measures of the product characteristics obtained in the measuring station it is possible to calculate the mean vector $\mu_{\mathrm{SP}_{1}}$ and the variance covariance matrix $\sum_{\mathrm{SP}_{1}}$ and thus, to realize a capability analysis with univariate PCIs for each product characteristic and with a multivariate PCI for the whole $\mathrm{SP}_{1}$.

The outputs of $\mathrm{SP}_{1}$ that have all their product characteristics within the specifications are described as conforming and are sent to $\mathrm{SP}_{2}$. Outputs with measures outside the SL are represented as nonconforming; and thus, cannot be sent to $\mathrm{SP}_{2}$. Nonconforming outputs cause additional production costs because these outputs must be reworked and tested again (and/or rejected) before being sent to $\mathrm{SP}_{2}$. The additional production costs due to rework are named rework costs.

In $\mathrm{SP}_{2}$ the products described as conforming after $\mathrm{SP}_{1}$ are processed. After it, the quality of the outputs is evaluated by analyzing other $n$ product characteristics: $\mathrm{PC}_{\mathrm{SP} 2,1}, \ldots, \mathrm{PC}_{\mathrm{SP} 2, \mathrm{n}}$. For each product characteristic $j$ of $\mathrm{SP}_{2}, \mathrm{PC}_{\mathrm{SP} 2, \mathrm{j}}$, $\mathrm{j} \in[1, \mathrm{n}] ; \mathrm{j} \in$ 
$\mathbb{N}$, the measured values of the $n$ product characteristics must be within $\mathrm{LSL}_{\mathrm{SP}_{2, \mathrm{j}}}$ and $\mathrm{USL}_{\mathrm{SP}_{2, \mathrm{j}}}$. Analogously to $\mathrm{SP}_{1}$, it is possible to do a capability analysis with univariate PCIs for each product characteristic and with a multivariate PCI for the whole $\mathrm{SP}_{2}$.

As it is possible to understand, the number of products that arrive to $\mathrm{SP}_{2}$ depends on the specification limits used in the quality test of $\mathrm{SP}_{1}$ : The smaller the distance between the specification limits in the quality test of $\mathrm{SP}_{1}$, the smaller the proportion of conforming parts in $\mathrm{SP}_{1}$; and thus, the smaller the proportion of parts that is sent to $\mathrm{SP}_{2}$. For this reason, we can affirm that the proportion of nonconforming parts in $\mathrm{SP}_{1}$ and $\mathrm{SP}_{2}$ depends on the values of the specification limits of $\mathrm{SP}_{1}$. Taking it into account, we suggest obtaining the optimal values of the specification limits of $\mathrm{SP}_{1}$ that minimize all rework costs of the multistage production process.

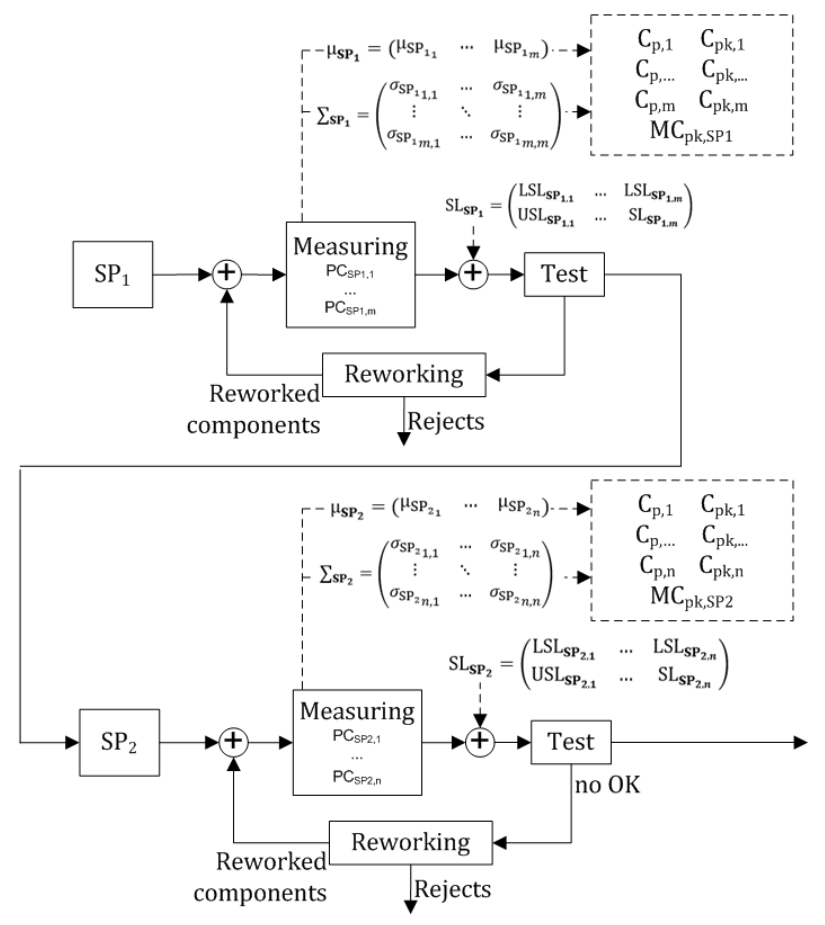

Fig. 1. Multivariate and multistage production process with two stages.

Minimizing all rework costs means that minimizing the sum of the proportion of nonconforming outputs of each subprocess weighted by the unitary reworking costs. The unitary reworking costs describe the rework costs originated when reworking an output. Taking all this into account, we propose the following MRO model:

$$
\begin{aligned}
& \text { [MIN] } k_{S P_{1}} \cdot N C P_{S P_{1}}+k_{S P_{2}} \cdot N C P_{S P_{2}} \\
& N C P_{S P_{1}}=1-\int_{S R_{1}} \frac{1}{\sqrt{\left|\sum \mathrm{SP}_{1}\right|(2 \pi)^{m}}} e^{-\frac{1}{2}\left(x_{1}-\mu_{1}\right)^{\prime} \sum \mathrm{SP}_{\mathbf{1}}{ }^{-1}\left(\boldsymbol{x}_{\mathbf{1}}-\mu_{\mathbf{1}}\right)} d S R_{1} \\
& N C P_{S P_{2}}=N C P_{S P_{T}}-N C P_{S P_{1}} \\
& N C P_{S P_{T}}=1-\int_{S R_{T}} \frac{1}{\sqrt{\left|\sum \mathbf{S P}_{T}\right|(2 \pi)^{m+n}}} e^{-\frac{1}{2}\left(x_{T}-\mu_{T}\right)^{\prime} \sum \mathbf{s p}_{T}^{-1}\left(x_{T}-\mu_{T}\right)} d S R_{T}
\end{aligned}
$$

$$
\begin{array}{cc}
L S L_{1_{i, i n i t}} \leq L S L_{1_{i}} \leq T_{1_{i}} & \forall i \in[1, m] \\
T_{1_{i}} \leq U S L_{1_{i}} \leq U S L_{1_{i, i n i t}} & \forall i \in[1, m]
\end{array}
$$

where:

$k_{S P_{1}}$ and $k_{S P_{2}}$ are the unitary reworking costs that describe the rework cost of each nonconforming output of $\mathrm{SP}_{1}$ and $\mathrm{SP}_{2}$. They are measured in monetary units / output.

$N C P_{S P_{1}}, N C P_{S P_{2}}$ and $N C P_{S P_{\mathrm{T}}}$ are the proportion of nonconforming parts of $\mathrm{SP}_{1}, \mathrm{SP}_{2}$ and of the whole multistage production process.

$\boldsymbol{\mu}_{1}=\left(\mu_{1,1}, \ldots, \mu_{1, i}, \ldots, \mu_{1, m}\right)$ is the mean vector that includes the mean values of the $m$ measured product characteristics of $\mathrm{SP}_{1}$.

$\boldsymbol{x}_{\mathbf{1}}=\left(x_{1,1}, \ldots, x_{1, i}, \ldots, x_{1, m}\right)$ are the integration variables in (6).

$\sum \mathbf{S P}_{\mathbf{1}}$ is the variance-covariance matrix $(m \times m)$ of the $m$ measured product characteristics of $\mathrm{SP}_{1}$.

$\boldsymbol{\mu}_{\mathbf{T}}=\left(\mu_{T_{1}}, \ldots, \mu_{T_{k}}, \ldots, \mu_{T_{m+n}}\right)$ is the mean vector that includes the mean values of the $m+n$ measured product characteristics in $\mathrm{SP}_{1}$ and in $\mathrm{SP}_{2} . k \in[1, \mathrm{n}+\mathrm{m}] ; k \in \mathbb{N}$. It is important to notice that the first $m$ components in $\boldsymbol{\mu}_{\mathrm{T}}$ represent the mean values of the $m$ product characteristics of $\mathrm{SP}_{1}$ and the last $n$ components in $\boldsymbol{\mu}_{\mathbf{T}}$ represent the mean values of the $n$ product characteristics of $\mathrm{SP}_{2}$.

$\boldsymbol{x}_{\mathbf{T}}=\left(x_{T, 1}, \ldots, x_{T, k}, \ldots, x_{T, m+n}\right) \quad$ are the integration variables in (8).

$\sum \mathbf{S P}_{\boldsymbol{T}}$ is the variance-covariance matrix $(m+n) \times(m+n)$ of the $m+n$ estimated product characteristics. It is important to notice that the submatrix $(m \times m)$ placed on the first $m$ columns and rows is the variance covariance matrix of $\mathrm{SP}_{1}$, $\sum_{\mathbf{S P}_{\mathbf{1}}}$. Analogously, the submatrix $(n \times n)$ placed on the last $n$ columns and rows is the variance-covariance matrix of $\mathrm{SP}_{2}$, $\sum \mathrm{SP}_{2}$.

$\mathbf{S R}_{\mathbf{1}}$ is the specification region for $\mathrm{SP}_{1}$ delimited by $\mathbf{S L}_{\mathbf{S P}_{\mathbf{1}}}(2 \times m) . \mathbf{S L}_{\mathbf{S P}_{\mathbf{1}}}$ is the specification limit matrix of $\mathrm{SP}_{\mathbf{1}}$ which includes in columns the LSL and the USL of each product characteristic $i, \forall i \in[1, m]$.

$$
\mathbf{S L}_{\mathbf{S P}_{1}}=\left(\begin{array}{ccccc}
\mathrm{LSL}_{\mathrm{SP}_{1,1}} & \cdots & \mathrm{LSL}_{\mathrm{SP}_{1, \mathrm{i}}} & \cdots & \mathrm{LSL}_{\mathrm{SP}_{1, \mathrm{~m}}} \\
\mathrm{USL}_{\mathrm{SP}_{1,1}} & \cdots & \mathrm{USL}_{\mathrm{SP}_{1, \mathrm{i}}} & \cdots & \mathrm{USL}_{\mathrm{SP}_{1, \mathrm{~m}}}
\end{array}\right)
$$

$\mathbf{S R}_{\mathbf{T}}$ is the specification region for the whole multistage process delimited by $\mathbf{S L}_{\mathbf{T}}(2 \times(m+n))$. $\quad \mathbf{S L}_{\mathbf{T}}$ is the specification limit matrix of the whole multistage process which includes in columns the LSL and the USL of each $k[1, m+n]$ product characteristic in $\mathrm{SP}_{1}$ and $\mathrm{SP}_{2}$. The first $m$ columns of $\mathbf{S L}_{\mathbf{T}}$ are the columns of $\mathbf{S L}_{\mathbf{S P}_{\mathbf{1}}}$. The last $n$ columns of $\mathbf{S L}_{\mathbf{T}}$ are the columns of $\mathbf{S L}_{\mathbf{S P}_{2}}$.

$$
\boldsymbol{S L}_{\mathrm{T}}=\left(\begin{array}{ccccc}
\mathrm{LSL}_{\mathrm{T}_{1}} & \cdots & \mathrm{LSL}_{\mathrm{T}_{\mathrm{k}}} & \cdots & \mathrm{LSL}_{\mathrm{T}_{\mathrm{m}+\mathrm{n}}} \\
\mathrm{USL}_{\mathrm{T}_{1}} & \cdots & \mathrm{USL}_{\mathrm{T}_{\mathrm{k}}} & \cdots & \mathrm{USL}_{\mathrm{T}_{\mathrm{m}+\mathrm{n}}}
\end{array}\right)
$$

$\mathrm{LSL}_{1_{i} \text {,init }}$ and $\mathrm{USL}_{1_{\mathrm{i} \text {,init }}}$ are the initial values of the LSL and the USL of each product characteristic $i \in[1, m]$ from $\mathrm{SP}_{1}$. 
$\mathrm{T}_{1_{\mathrm{i}}}$ are the $i$ target values of each product characteristic $i \in[1, m]$ from $\mathrm{SP}_{1}$.

It is important to realize that minimizing the rework costs of the whole multistage production process by modifying the configuration of the specification limits in the first sub-process will provoke the appearance of additional nonconforming outputs (ANCOs) in the first sub-process. ANCOs are outputs described as nonconforming when using the optimal configuration of the specification limits although they were described as conforming when using the original configuration of the specification limits.

\section{AN EXAMPLE}

In this section we are going to apply the proposed optimization model in an example based on a production process of the automotive sector. The production process that is going to be analyzed is a multistage production process with two sub-processes. The first sub-process is the machining process of an engine component. The second sub-process is the assembling process of the engine. This multistage production process can be represented using the same representation used in Fig. 1.

In the machining sub-process $\left(\mathrm{SP}_{1}\right)$ there are two product characteristics that are the focus of study $(m=2)$. The first product characteristic is named $\mathrm{PC}_{\mathrm{SP} 1,1}$. The LSL of $\mathrm{PC}_{\mathrm{SP} 1,1}$ (LSL $\mathrm{LP}_{1,1}$ ) is placed on $-1.00 \mathrm{~mm}$ and the USL of $\mathrm{PC}_{\mathrm{SP} 1,1}\left(\mathrm{USL}_{\mathrm{SP} 1,1}\right)$ is placed at $1.00 \mathrm{~mm}$. The target value $\left(\mathrm{T}_{\mathrm{SP} 1,1}\right)$ is placed on the middle point: $0.00 \mathrm{~mm}$. The second product characteristic is named $\mathrm{PC}_{\mathrm{SP} 1,2}$. The LSL of $\mathrm{PC}_{\mathrm{SP} 1,2}$ $\left(\mathrm{LSL}_{\mathrm{SP} 1,2}\right)$ is placed on $-1.00 \mathrm{~mm}$. The USL of $\mathrm{PC}_{\mathrm{SP} 1,2}$ (USL $\left._{\mathrm{SP} 1,2}\right)$ is placed on $1.00 \mathrm{~mm}$. The target value $\left(\mathrm{T}_{\mathrm{SP} 1,2}\right)$ is also placed at the middle point: $0.00 \mathrm{~mm}$.

In the assembling sub-process $\left(\mathrm{SP}_{2}\right)$ there are also two product characteristics that are the focus of study $(n=2)$. The first product characteristic is named $\mathrm{PC}_{\mathrm{SP} 2,1}$ and the second is named $\mathrm{PC}_{\mathrm{SP} 2,2}$. $\mathrm{LSL}_{\mathrm{SP} 2,1}$ is placed at $-1.00 \mathrm{~mm}$ and $\mathrm{USL}_{\mathrm{SP} 2,1}$ is placed at $1.00 \mathrm{~mm}$. The target value $\left(\mathrm{T}_{\mathrm{SP} 2,1}\right)$ is placed at the middle point: $0.00 \mathrm{~mm}$. $\mathrm{LSL}_{\mathrm{SP} 2,2}$ is placed at $-1.00 \mathrm{~mm}$ and $\mathrm{USL}_{\mathrm{SP} 2,2}$ is placed at $1.00 \mathrm{~mm}$. The target value $\left(\mathrm{T}_{\mathrm{B}}\right)$ is placed at the middle point: $0.00 \mathrm{~mm}$.

We generate a 4-variate sample of data with 10.000 points based on a production process of the automotive industry and we assume that reworking an output in the assembly lines is 5 times more expensive as reworking it in the machining lines $\left(k_{S P_{1}}=1 € /\right.$ unit and $k_{S P_{2}}=5 € /$ unit $)$.

Before applying the MRO model from section III, we want to show the influence of the values of the specification limits of $\mathrm{SP}_{1}$ in the total rework costs. Fig. 2 shows six contour plots. Each contour plot is determined by the specification limits of $\mathrm{SP}_{1}$ and represents the minimal achievable rework costs for the whole process (also considering the two specification limits that are not represented in the axes) by considering the initial sample of data. We can see that the rework costs, which must be assumed in this multistage production process, depend on the values of the specification limits of the first sub-process.
We can observe that the smaller the specification region (SL near the target value), the higher the rework costs. Furthermore, we can observe that the configuration of the specification limits that minimize rework costs is near the initial specification limits. Hereafter, we apply the MRO model from section III to obtain a more precise configuration of the optimal specification limits.
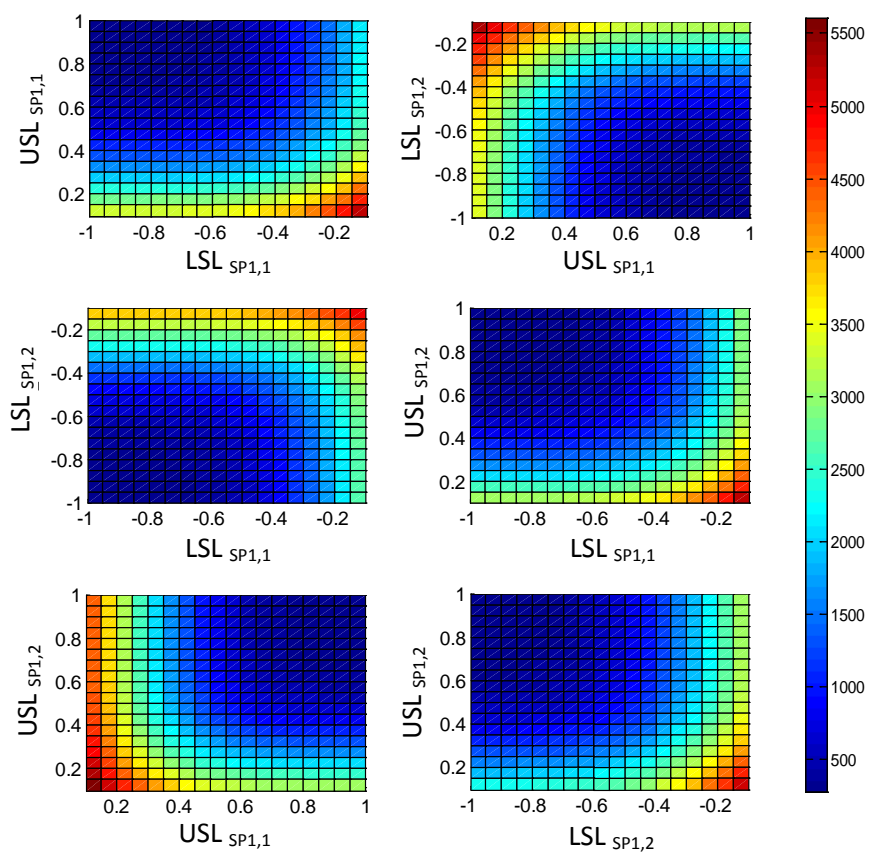

Fig. 2. Rework costs (in $€$ ) depending on the configuration of the specification limits in the machining process.

In order to apply the MRO model from section III, we need to obtain the mean vectors (13) and (14); and the variancecovariance matrices (15) and (16) from the sample.

$$
\begin{gathered}
\boldsymbol{\mu}_{\mathbf{1}}=\left(\begin{array}{lll}
0.039 & -0,060
\end{array}\right) \\
\boldsymbol{\mu}_{\mathrm{T}}=\left(\begin{array}{llll}
0.039 & -0,060 & 0.244 & 0.305
\end{array}\right) \\
\sum \mathbf{S P}_{\mathbf{1}}=\left(\begin{array}{lll}
0.089 & 0.022 \\
0.022 & 0.091
\end{array}\right) \\
\sum_{\mathbf{S P}_{T}}=\left(\begin{array}{rrrr}
0.089 & 0.022 & 0.064 & -0.021 \\
0.022 & 0.091 & 0.020 & 0.055 \\
0.064 & 0.020 & 0.087 & 0.013 \\
-0.021 & 0.055 & 0.013 & 0.086
\end{array}\right)
\end{gathered}
$$

This example has been solved with MATLAB R2013b using a workstation with CPU Intel $\AA^{\circledR}$ Core $^{\mathrm{TM}}$ i5-5300U CPU @2.30 GHz and runtime of 61.25s. The solver function patternsearch in MATLAB was used. The optimal values of the specification limits of $\mathrm{SP}_{1}$ are tabulated in table I.

If we look at table I, we can see that the optimal LSLs for $\mathrm{PC}_{\mathrm{SP} 1,1}$ and $\mathrm{PC}_{\mathrm{SP} 1,2}$ are nearly the same as the initial ones. For the USLs, the MRO model suggests two different values. If we refine and adjust the mesh used in Fig. 2, we can see that the optimal values obtained for both upper specification limits are in accordance with the values suggested by the model (see Fig. 3). 
In table I we can also see that the rework costs of the whole multistage production process have been reduced. The initial configuration of the specification limits carries a cost of $661 €$. The optimal configuration reduces the unitary costs to $642 €$, which is a reduction of $2.87 \%$ of the rework costs.

TABLE I. COMPARISON OF INITIAL AND OPTIMAL CONFIGURATIONS ${ }^{\mathrm{a}}$

\begin{tabular}{|c|c|c|c|c|c|}
\hline Configuration & $\mathbf{L S L}_{\mathbf{S P 1} \mathbf{1}}$ & $\mathbf{U S L}_{\mathbf{S P 1} \mathbf{1} \mathbf{1}}$ & $\mathbf{L S L}_{\mathbf{S P 1} \mathbf{2}}$ & $\mathbf{U S L}_{\mathbf{S P 1} \mathbf{2}}$ & $\mathbf{R C}$ \\
\hline Initial & -1 & 1 & -1 & 1 & 661 \\
\hline Optimal & -0.98 & 0.88 & -1.00 & 0.72 & 642 \\
\hline
\end{tabular}

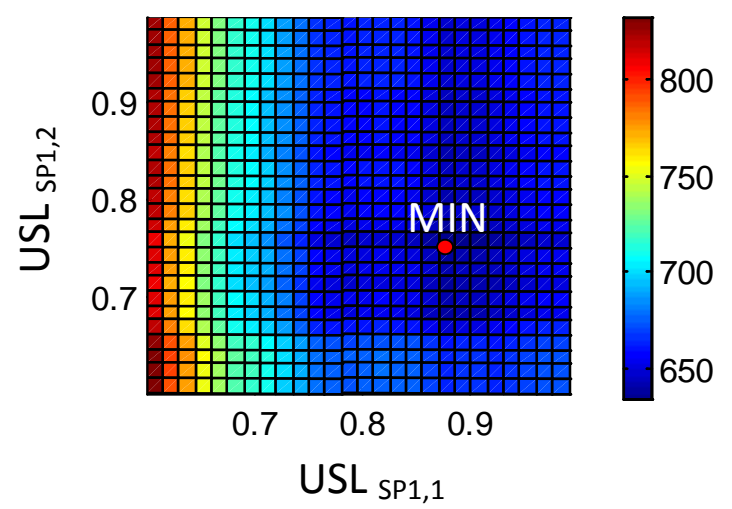

Fig. 3. Refined and adjusted mesh for the entire rework costs (in €) depending on the configuration of the USL $L_{S P 1,1}$ and USL $L_{S P 1,2}$ in the machining process.

Hereafter we analyze how the optimal configuration of the specification limits affects the capability of this multistage production process. In table II we can find the univariate PCIs for the four product characteristics analyzed in this example for the initial and the optimal configuration of the specification limits.

TABLE II. UNIVARIATE CAPABILITY ANALYSIS

\begin{tabular}{|c|c|c|c|c|c|}
\hline Configuration & $\mathbf{P C I}$ & $\mathbf{P C}_{\mathbf{S P 1} \mathbf{1}}$ & $\mathbf{P C}_{\mathbf{S P 1} \mathbf{2}}$ & $\mathbf{P C}_{\mathbf{S P 2} \mathbf{1}}$ & $\mathbf{P C}_{\mathbf{S P} \mathbf{2} \mathbf{2}}$ \\
\hline \multirow{3}{*}{ Initial } & $\mathrm{C}_{\mathrm{p}}$ & 1.12 & 1.11 & 1.13 & 1.14 \\
\cline { 2 - 6 } & $\mathrm{C}_{\mathrm{pk}}$ & 1.07 & 1.04 & 0.86 & 0.79 \\
\hline \multirow{3}{*}{ Optimal } & $\mathrm{C}_{\mathrm{p}}$ & 1.04 & 0.95 & 1.14 & 1.15 \\
\cline { 2 - 6 } & $\mathrm{C}_{\mathrm{pk}}$ & 0.93 & 0.86 & 0.86 & 0.80 \\
\hline
\end{tabular}

With the optimal configuration, the capability regarding the product characteristics of the machining process $\left(\mathrm{PC}_{\mathrm{SP} 1,1}\right.$ and $\mathrm{PC}_{\mathrm{SP} 1,2}$ ) has been reduced. This occurs due to the fact that the optimal USLs of both product characteristics is smaller than the original one; and thus, the probability of finding nonconforming outputs in the optimal configuration is higher than in the initial configuration. However, the capability regarding the product characteristics of the assembly lines increases. This occurs because many of the nonconforming outputs that were detected in the test of the assembly lines in the initial configuration are detected in the test of the machining process with the optimal configuration; and thus, they are not sent to the assembly sub-process.

Table III shows the multivariate PCIs for $\mathrm{SP}_{1}$ and $\mathrm{SP}_{2}$ for the initial and the optimal configurations. We can see that the optimal configuration reduces the capability of the machining process but increases the global capability of the assembly process. If we consider the capability of the whole multistage process, we can see that it presents lower capability in the optimal configuration. This happens because taking into account the rework costs of the whole multistage process, the probability of describing outputs as nonconforming in the optimal configuration of the specification limits is higher. With the proposed MRO model, the total rework costs are minimized but we have to accept smaller rates of conforming parts and the appearance of ANCOs.

TABLE III. MULTIVARIATE CAPABILITY ANALYSIS

\begin{tabular}{|c|c|c|c|c|}
\hline Configuration & PCI & $\mathbf{S P}_{\mathbf{1}}$ & $\mathbf{S P}_{\mathbf{2}}$ & $\mathbf{S P}_{\mathbf{T}}$ \\
\hline Initial & $\mathrm{MC}_{\mathrm{pk}}$ & 0.99 & 0.73 & 0.72 \\
\hline Optimal & $\mathrm{MC}_{\mathrm{pk}}$ & 0.81 & 0.74 & 0.69 \\
\hline
\end{tabular}

Finally, we want to analyze the appearance of ANCOs because of the optimal configuration of the specification limits in this example. Table IV shows the engines described as nonconforming in $\mathrm{SP}_{1}, \mathrm{SP}_{2}$ and $\mathrm{SP}_{\mathrm{T}}$ for the initial and the optimal configurations. The difference between the outputs described as nonconforming for $\mathrm{SP}_{\mathrm{T}}$ in the optimal and in the initial configuration are the ANCOs. As stated, ANCOs are outputs that should be described as conforming but with the proposed configuration of the specification limits are described as nonconforming. ANCOs will not leave the multistage production process; and thus, they generate a loss of benefit.

TABLE IV. ANCOS ANALYSIS

\begin{tabular}{|c|c|c|c|}
\hline Nonconforming outputs & $\mathbf{S P}_{\mathbf{1}}$ & $\mathbf{S P}_{\mathbf{2}}$ & $\mathbf{S P}_{\mathbf{T}}$ \\
\hline Initial & 20 & 129 & 149 \\
\hline Optimal & 72 & 114 & 186 \\
\hline
\end{tabular}

Hereafter, we evaluate the value of the model by comparing the rework costs that must be assumed in a second scenario with a new sample of data of 10.000 points. Table $\mathrm{V}$ shows the rework costs that must be assumed with the initial configuration of the specification limits and the optimal configuration obtained in this section.

We can see that using the optimal configuration of the specification limits, the rework costs are smaller than the rework costs that should be assumed with the initial configuration. The initial configuration of the specification limits carries a rework cost of $713 €$. The optimal configuration reduces the costs to $673 €$, which is a reduction of $5.61 \%$ of the rework costs.

TABLE V. COMPARISON OF INITIAL AND OPTIMAL CONFIGURATIONS WITH A SECOND SAMPLE ${ }^{\mathrm{b}}$ 


\begin{tabular}{|c|c|c|c|c|c|}
\hline Configuration & LSL $_{\mathbf{S P} \mathbf{1} \mathbf{1}}$ & $\mathbf{U S L}_{\mathbf{S P} \mathbf{1} \mathbf{1}}$ & $\mathbf{L S L}_{\mathbf{S P} \mathbf{1}, \mathbf{2}}$ & $\mathbf{U S L}_{\mathbf{S P} \mathbf{1} \mathbf{2}}$ & $\mathbf{R C}$ \\
\hline Initial & -1 & 1 & -1 & 1 & 713 \\
\hline Optimal & -0.98 & 0.88 & -1.00 & 0.72 & 673 \\
\hline
\end{tabular}

[3] E. Lee, “Cyber physical systems: Design challenges,” 2008 11th IEEE International Symposium on Object Oriented Real-Time Distributed Computing (ISORC), pp. 363-369, 2008.

We can see that the optimal configuration of the specification limits derived from the first sample is also valid for the second sample. This occurs because production parameters have not varied between the first and the second samples; and thus, the second sample has the same mean vector and variance-covariance matrix as the first sample.

\section{CONCLUSIONS AND OUTLOOK}

In this paper a MRO model has been presented. This MRO model differs from other MRO models from the literature in three aspects: First, the model uses the modularity derived from multistage production processes by considering each stage individually and afterwards the whole multistage process as a global system. Second, in the presented model, rework costs are taken into account. Third, in the presented model, the optimization variables are the specification limits. With this model, rework costs originated due to poor production processes can be easily minimized when machines cannot be recalibrated. While minimizing the whole rework costs using the proposed model, the proportion of outputs of the whole process that will be described as nonconforming will increase; and consequently, more outputs will have to be reworked. Thus, if companies want to minimize rework costs using the proposed MRO model, they will have to be able to assume more rework rates as well as ANCOs. It has been seen that it is possible to minimize quickly rework costs of a multistage production process when sacrificing capability in the first subprocesses.

For future research we propose improving the MRO model with focus on these three points: Firstly, the proposed model assumes that the specification limits of the last stage of the multistage production process are fixed. If the specification limits in the last stage are also flexible, we suggest using the proposed model with different configurations of the specification limits in the last stage of the process. Then, we suggest obtaining the optimal specification limits in the first stage for each configuration and taking the configuration that carries the minimal rework costs. This may enable researchers to minimize rework costs in the case where the specification limits of the last stage are not fixed. Secondly, the presented model does not take into account the loss originated by the ANCOs. Thus, we recommend to include the cost originated by the ANCOs in the model. Finally, future researchers may also deal with the case in which the measures are not normal distributed.

\section{REFERENCES}

[1] CPS Steering Group, "Cyber-physical systems executive summary," CPS Summit 2008, 2008.

[2] N. Jazdi, “Cyber physical systems in the context of industry 4.0," 2014 IEEE International Conference on Automation, Quality and Testing, Robotics, 2014.

[5] Y. Yu, Y. Chen, Y. Bi and F. Chai, "Adaptive Control of NegativeSaliency PMSM based on Online Parameter Identification,” 2016 IEEE 42nd Annual Conference of Industrial Electronics Society, 2016.

[6] E. Harrington, "The Desirability Function,” Industrial Quality Control, vol. 21, num. 10, pp. 494-498, 1965.

[7] G. Derringer and R. Suich, "Simultaneous Optimization of Several Response Variables," Journal of Quality Technology, vol. 12, num. 4, pp. 214-219, 1980.

[8] E. del Castillo, D.C. Montgomery and D.R. McCarville, "Modified desirability functions for multiple response optimization," Journal of Quality Technology, vol. 28, pp. 337-345, 1996.

[9] K.J. Kim and D.K.J Lin, "Simultaneous optimization of mechanical properties of steel by maximizing exponential desirability functions," Journal of the Royal Statistical Society: Series C (Applied Statistics), vol. 49, num. 3, pp.311-325, 2000.

[10] C.F.J. Wu, M.S. Hamada, Experiments: Planning, Analysis, and Parameter Design Optimization, vol. 552. John Wiley \& Sons, 2011.

[11] J.J. Pignatiello, "Strategies for robust multiresponse quality engineering,” IIE Transactions, vol. 25, num. 3, pp. 5-15, 1993.

[12] G.G. Vining, "A compromise approach to multiresponse optimization," Journal of Quality Technology, vol. 30, num. 4, pp. 309-313, 1998.

[13] K.L. Tsui, "Robust design optimization for multiple characteristic problems," International Journal of Production Research, vol. 37, num. 2, pp. 433-445, 1999.

[14] J.L.D. Ribeiro, F.S. Fogliatto and C.S.T. Caten, "Minimizing manufacturing and quality costs in multiresponse optimization," Quality Engineering, vol. 13, num. 4, pp. 559-569, 2001.

[15] Y.H. Ko, K.J. Kim, and C.H. Jun, "A New Loss Function-Based Method for Multiresponse Optimization," Journal of Quality Technology, vol. 37, num. 1, pp. 50-59, 2005.

[16] L.I. Tong, C.H. Wang and H.C. Chen, "Optimization of multiple responses using principal component analysis and technique for order preference by similarity to ideal solution," International Journal of Advanced Manufacturing Technology, vol. 27, num. 3-4, pp. 407-414, 2005.

[17] H.C. Liao, "Multi-response optimization using weighted principal component," International Journal of Advanced Manufacturing Technology, vol. 27, num. 7-8, pp. 720-725, 2006.

[18] L.I. Tong, C.C. Chen and C.H. Wang, "Optimization of multiresponse processes using the VIKOR method," The International Journal of Advanced Manufacturing Technology, vol. 31, num. 11-12, pp. 10491057, 2007.

[19] S.K. Gauri and S. Chakraboroty, "Multi-response optimisation of WEDM process using principal component analysis," The International Journal of Advanced Manufacturing Technology, vol. 41, num. 7-8, pp. 741-748, 2009.

[20] C.H. Chiao and M. Hamada, "Analyzing experiments with correlated multiple responses,” Journal of Quality Technology, vol. 33, num. 4, pp. 451-465, 2001.

[21] J.J. Peterson, "A posterior predictive approach to multiple response surface optimization,” Journal of Quality Technology, vol. 36, num. 2, pp. 139-153, 2004.

[22] A.I. Khuri and M. Conlon, "Simultaneous optimization of multiple responses represented by polynomial regression functions," Technometrics, vol. 23, num. 4, pp. 363-375, 1981.

[23] P.H. Lee and B.J. Yum, "Multi-characteristics parameter design: a desirability function approach based on process capability indices," International Journal of Reliability, Quality and Safety Engineering, vol. 10, num. 4, pp. 445-461, 2003.

[24] M.I. Awad and J.V. Kovach, "Multiresponse optimization using multivariate process capability index," Quality and Reliability Engineering International, vol.24, num. 4, pp. 465-477, 2011. 
[25] A. Amiri, M. Bashiri, H. Mogouie and M.H. Doroudyan, "Non-normal multi-response optimization by multivariate process capability index," Scientia Iranica, vol. 19, num. 6, pp. 1894-1905, 2012.

[26] S. Bera and I. Mukherjee, "An integrated approach based on principal component and multivariate process capability for simultaneous optimization of location and dispersion for correlated multiple response problems,” Quality Engineering, vol. 25, num. 3, pp. 266-281, 2013.

[27] V.E. Kane, "Process capability indices,”Journal of Quality Technology, vol. 18, num.1, pp. 41-52, 1986.

[28] D. de-Felipe, T. Klee, J. Folmer, E. Benedito and B. Vogel-Heuser, “A multivariate process capability index that complies with industry requirements". 2016 IEEE 42nd Annual Conference of Industrial Electronics Society, 2016. 